\title{
Power - Aware Virtual Machine Consolidation considering Multiple Resources with Live Migration
}

\author{
Pallavi Gupta \\ CSE Department \\ Samrat Ashok Technological \\ Institute, Vidisha (MP), India
}

\author{
Lokendra Vishwakarma \\ CSE Department \\ Samrat Ashok Technological \\ Institute, Vidisha (MP), India
}

\author{
Awadheshwari Patel \\ CSE Department \\ Samrat Ashok Technological \\ Institute, Vidisha (MP), India
}

\begin{abstract}
Today the computing technologies require high level of efficiency and infrastructure that datacenters were unable to provide previously. Lack of efficient infrastructure can serve only limited number of users and simultaneously emits large amount of carbon footprint and over utilizes electrical power. Virtualization emerged as prime technique for service management and reduced energy expenses at datacenter. Hence, there is a need of virtualization techniques that enhance energy efficiency and CPU utilization along with required user's QoS (Quality of Service) in terms of minimum response time and maximum throughput. We propose efficient virtualization technique for dynamic VM consolidation according to the current power consumption by considering multiple resources (CPU, ram, and storage disk) with live migration of VMs by switching idle server to sleep mode, thus minimizing energy consumption at datacenter. The proposed approach can effectively handle strict SLA (Service Level Agreement) in homogeneous as well as in heterogeneous environment. The result verifies that the proposed algorithm reduces the number of active physical machines and it results into reduction of power consumption.
\end{abstract}

\section{Keywords}

Virtualization, VM Consolidation, Live Migration, Service Level Agreement, Energy Efficiency.

\section{INTRODUCTION}

Cloud Computing is basis for provisioning of computer resources to end user by pooling servers called as resources which are geographically distributed. User pays for services and applications that they are using for particular period. Consumers in cloud computing need not invest in building and maintaining complex IT infrastructure [1]. Large scale computing datacenter and expansion of high performance computing infrastructure consumes high electrical power which is harmful to environment [2].

According to report by Koomey there is continues increase in power consumption that has doubled itself from 2000 to 2005, representing annual growth of $14 \%$ in US only and $16 \%$ worldwide [2]. The total power consumption of all servers alone in US was $2.6 \times 10^{12}$ watt ( 2.6 billion $\mathrm{kw}$ ) in 2005 , while $7 \times 10^{12}$ watt worldwide. At the same time, the energy required for cooling the power ancillary equipment together uses $5.2 \times 10^{12}$ watt for US and $14 \times 10^{12}$ watt worldwide. Thereby IT enterprise is under the restriction imposed by regulation of government to go greener or use energy efficient technology. So it is a constant demand to reduce energy consumption to increase profitability and sustainability of the datacenter [3]. The major concern is how to reduce power consumption requirement of large IT infrastructure settings. The parameters like SLA (Service Level Agreement), power consumption, electricity cost have directly influenced the render services over cloud. Since datacenters are using large electrical power causing carbon footprint, it is very important for us to incorporate the energy efficiency resource management with SLA consideration for controlling electrical power consumption and reduction of carbon emission.

SLA (Service Level Agreement) is an agreement between user and service provider on the quality of service which has to be delivered to consumer and provider is bound to give required quality of service otherwise will be penalized [4]. Virtualization [5] technology allows one to create multiple Virtual Machines (VMs) on physical server and therefore reduces hardware requirement. It improves utilization of resources by using existing hardware. The advantage of virtualization is to improve fault and performance isolation between application sharing the same resources, ability to migrate VMs from one physical host to another using live migration and to support for hardware and software heterogeneity.

Main issue that needs to be resolved is that which virtual machine has to be consolidated at which physical server. This decision has direct impact on power consumption of host. The indicated problem is resolved by developing efficient energy conscious provisioning policy which can be implied to the resources and application level virtualization. Server consolidation allows cloud service vendor to increase CPU utilization by binding multiple virtual machine into limited number of physical server, thus bring down electricity charges. The binding is done by migrating virtual machines from one host to any other host [5]. Reducing the number of physical server by switching off the idle server can save huge amount of electrical power and maintenance cost because it is estimated in the "server energy and efficiency report" that 10 to $15 \%$ servers are 'ghost servers' [6]. Ghost servers are those that consume $70 \%$ of the total power at their idle state which is equivalent to power consumed by any server at $100 \% \mathrm{CPU}$ utilization. Author states that usually server is utilized only for $30 \%$ of their utilization time while they are idle for rest of the period causing huge wastage of electrical power and money [7].

The rest of the paper is organized as follows: Section 2 discusses related works. Section 3 presents the system architecture and the details of the proposed algorithm. Section 4 demonstrates experimental results and Conclusions and future work are finally given in Section 5 .

\section{RELATED WORK}

Virtualization is the use of hardware and software resources to create the perception that one or more entities exist, although the entities are not physically exist. Using virtualization, we allow multiple operating systems to run on single computer 
system. Calheiros et al. described high and peak loads on cloud resources (such as datacenters, host machines, storage) may over heat them, this leads to the reduction in system reliability and lifetime [8]. Efficient energy conscious provisioning policy can solve this problem.

Initially, life span of mobile batteries was improved by energy aware resource management techniques [9] [10]. Later thi context has been applied to datacenter [11] and virtual computing environment i. e. Cloud. In DVFS, dynamic manipulation of input voltage and clock frequency of CPU will reduce power consumption [12]. Earlier power minimizing algorithm uses dynamic switching of server mode between ON and OFF state whenever new request arrives [13]. Cole defined the resource provisioning framework in which resource provisioning is done power efficiently as well as ensures that by using workload forecasting the user defined SLA meets the least Power consumption [6]. However, parameters are defined for homogeneous node and consider only single application workload. Learning from this idea and we propose our work for the heterogeneous environment.

Most of the existing consolidation methods proposed that VM is rigid entity i.e. VMs resource capacity remains unchanged during VM consolidation [14] [15]. Hu et al. develops 'squeeze' and 'release' measure to dynamically redistribute workload on each node according to requirement on each node in cluster environment [16]. VM migration is achieved by work load redistribution and idle node, not performing any task is switched off. However, this work focuses only on single resource which is CPU. In our proposed work, consolidation strategy considers multiple types of resources. Elnozahy et al. have investigated the problem of efficient management of resources in a single web application environment with fixed SLA (in terms of response time) [17].

Cardosa et al. [18] have proposed an approach for problem of power-aware allocation of VMs on virtualized heterogeneous system environment. They have min, $\max$ and share parameter of VMM (Virtual Machine Monitor) which represent minimum, maximum and proportion of the CPU allocated to VM sharing same resources. However, the approach is applicable in enterprise environment, as it does not support strict SLA and in this the share parameter depends on prior knowledge of application priorities and its limitation is that no other resource except CPU is considered during VM reallocation.

To achieve energy efficiency cloud computing present following methods:
1. VM Consolidation
2. VM migration
3. Switching On /Off server when required
4. Power aware resource allocation strategies
5. Replacing inefficient hardware

Liao et al. proposed an energy efficient resource provisioning technique for virtual machine scheduling [20]. They considered multiple resources for VM consolidation aiming to choose minimum number of physical machine. They have been shown that multiple resources in scheduling techniques will give minimization of power consumption with satisfying user's SLA request. The negative aspect of the approach is that live migration was not included. They consolidate VM only, when a new request is admitted and not implement any VM optimization during the processing of the request.

In contrast to this work, we propose efficient technique for dynamic VM consolidation according to current power consumption by resources like CPU, ram and disk with live migration. Admissions of new request are done by VM consolidation technique then optimize VMs by applying live migration. The migration overhead (in terms of migration time) will lead to performance degradation. To overcome this performance degradation, we use double threshold minimization of migration policy. This policy limits the number of migration as well as consumes minimum energy. After VM optimization, if there is any idle server i.e. not doing any useful work then switch them to sleep state and therefore minimizes energy consumption. The proposed approach can effectively handle strict SLA and effectively work with heterogeneous infrastructure.

\section{SYSTEM OVERVIEW}

In our proposed work, system architecture is based on the Green Cloud Computing. Green cloud computing is envisioned to achieve efficient processing and utilization as well as focuses on minimizing energy consumption [1]. In this approach, cloud datacenter comprises ' $n$ ' heterogeneous physical nodes. Each node has a CPU, with different number of processors, performance defined in terms of MIPS (Million Instructions per Second), storage (in GB) and RAM (in MB).

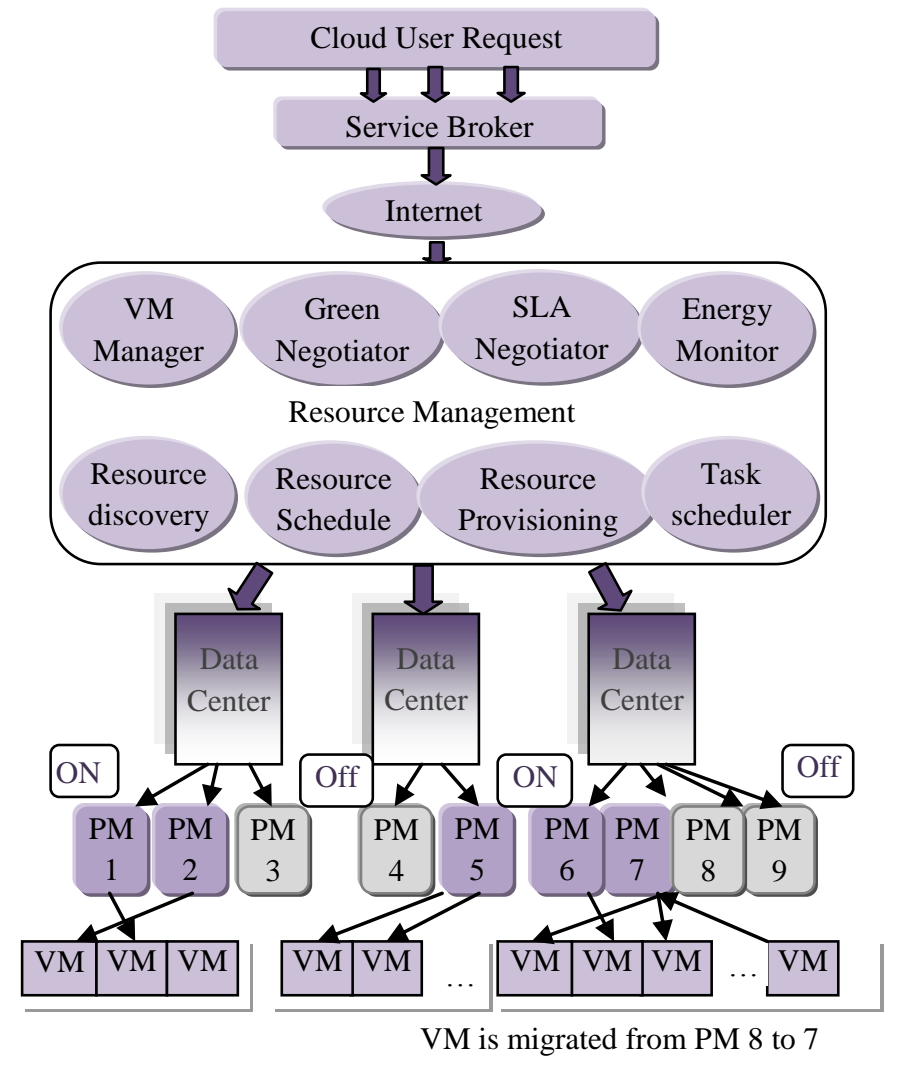

Fig. 1: System architecture

The Working of proposed system architecture is as follows:

1. User submits request (in cloudsim [8] a cloud user request is called as 'cloudlet request') for provisioning of VM.

2. 'Service Broker' receives cloudlets through internet and dispatches it to the Resource Management layer.

3. 'Green negotiator' will negotiate with the consumer/brokers to finalize the SLA and penalties between cloud provider and consumer depending on consumer's QoS requirements and energy saving schemes. 
4. In the 'resource discovery', user job request matches with available cloud resources.

5. The request is allocated to most energy efficient node considering multiple resources with 'resource provisioning policy'.

6. The VMs are consolidated to minimum number of host.

7. During VM optimization, VM Manager issues the Migration command again aiming to choose minimum number of host capable of accepting migration request thus increasing the CPU utilization of these hosts. Then switching OFF idle server.

Some Research challenges are:

1. How to decide, which VM has to be migrated on which host in order to reduce power consumption?

2. With applying migration, overhead is incurred in terms of migration time. To come across the problems which degrade performances and finally to solve the problem optimally i.e. the trade-off between energy saving and performance.

\section{Basic terminologies are:}

\subsection{SLA violation}

When the demand of CPU performance exceeds the existing capacity, a violation of the SLA's established between the resource provider and user. An SLA violation results in a penalty incurred to the provider. SLA can be measured in terms of throughput and response time. SLA violation can be measured with the two parameters.

1. The percentage of time, during which active hosts experienced $100 \%$ CPU utilization.

2. The overall performance degradation due to migration.

\subsection{VM provisioning}

It is allocation of $\mathrm{VM}$ to a particular request for processing and can be carried out in two steps.

1. In the first step, new request is admitted to host by using VM allocation policy. Our proposed VM allocation policy consolidate VMs to the energy efficient node while uses minimum number of host.

2. Second step is the optimization of current VM allocation. This is carried out using VM live migration. This consider as 'live' because VM continues its processing even during migration. VM migration is carried out in 3 steps.

\subsubsection{When to Migrate:}

VM migration can be performed to fulfill two objectives:

1. To balance load, if host is over utilized then migrate VMs to remaining underutilized host.

2. In VM consolidation to save energy, migrate VMs in order to choose minimum number of host and then Power OFF the remaining host.

Our objective is to reduce power consumption, minimizing performance degradation due to migration and simultaneously fulfilling the required QoS. Fig.2 represents the two scenarios where migration can be applied. There are 3 Physical Machines (PMs) having different number of virtual machines. First scenario represents the load balancing among 3 PMs. In this, one VM gets migrated from PM1 to PM3 aiming to balance load among all PMs. Second scenario represents the consolidation of VM into minimum number of PM in order to save energy and increase utilization of CPU. For detecting overload and under-load condition we employ double threshold policy. In this work, we take lower and upper threshold value $30 \%$ and $70 \%$ respectively. By keeping these threshold values, we try to optimally solve the trade-off between energy saving and performance. These threshold values limit the number of migration to control the performance degradation due to migration as well as minimize energy consumption.

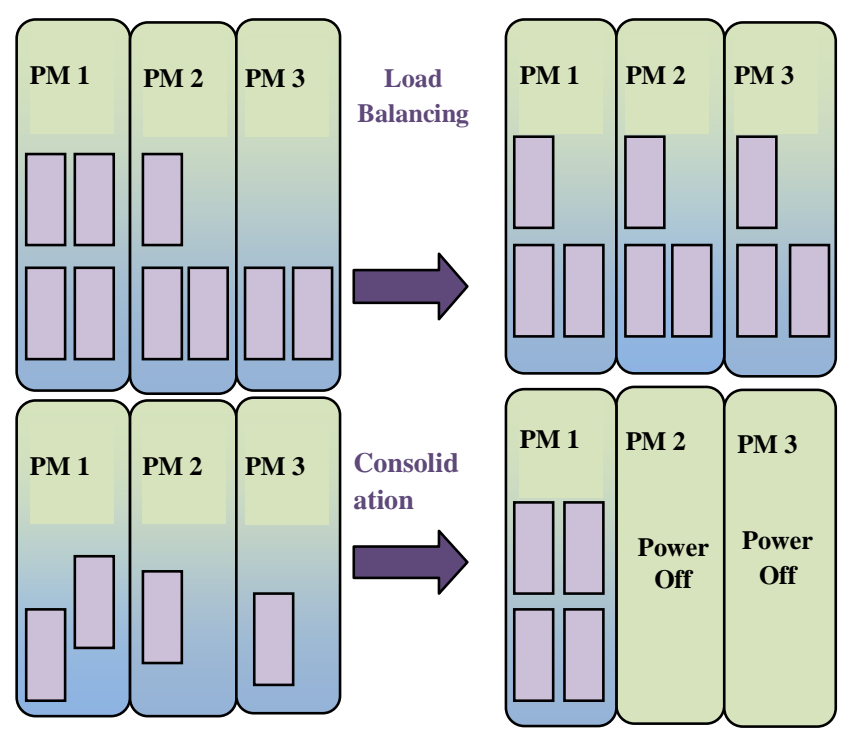

Fig. 2: Migration used in Load Balancing and

\section{Consolidation}

\subsubsection{Which VM to Migrate:}

This is done by VM selection policy. It is used to select particular VM to migrate from this host to another host. There are some policies for VM selection, namely:

1. The Random Choice Policy: randomly selects the VMs to migrate.

2. The Minimum Utilization Policy: selects those VM that have the lowest usage of CPU.

3. The Minimum Migration Time Policy: This policy migrate a VM that requires the minimum time to complete a migration compared to other VMs. Here migration time is calculated as the amount of available RAM divided by available spare network bandwidth for host. Here assumption is that all host having the same network bandwidth.

$$
\text { Migration time of a } \operatorname{Vm}(\mathrm{i})=R A M(i) / N E T(j)
$$

Where RAM (i) is the amount of RAM currently utilized by $\mathrm{Vm}$ (i) and NET (j) is the spare network bandwidth of host $\mathrm{j}$. The $\mathrm{Vm}$ with minimum migration time is selected for migration.

4. Minimization Of Migration: The MM (Minimization of Migration) policy migrate the minimum number of VMs. In this policy, VMs are migrated to other host when they have resource shortage such as when requested amount of processing speed (MIPS) is greater than available one.

In our work, we choose the minimization of migration policy for minimizing performance degradation due to migration. 


\subsubsection{Where to Migrate:}

VM placement policy selects the target host at which VM has to place after migration. The targeted host should not be overloaded after migration. We choose the most power efficient host for VM placement with the condition that it becomes not overloaded after migration.

In order to obtain the most energy efficient host for allocating VMs we choose host with the minimum value, i.e.

Min $\left\{\frac{\mathrm{un}_{\mathrm{cpu}_{\mathrm{i}}}-\mathrm{r}_{\mathrm{cpu}_{\mathrm{j}}}}{\text { Total }_{\text {cpu }_{i}}} * a+\frac{u_{\text {mem }_{i}}-r_{\text {mem }_{j}}}{\text { Total }_{\text {mem }_{i}}} * b+\frac{\text { un }_{\text {disk }_{i}}-r_{\text {disk }_{j}}}{\text { Total }_{\text {disk }}}\right.$ $\left.* c\right\}$ ......(1)

Here $\mathrm{un}_{-} \mathrm{cpu}_{\mathrm{i}}$ is the no. of unused Processing cores in a host $\mathrm{i}$ and it is calculated as

un_cpu $\mathrm{i}_{\mathrm{i}}=$ Total_cpu $\mathrm{i}_{\mathrm{i}}-$ Static_cpu $\mathrm{c}_{\mathrm{i}}-\sum_{j=1}^{N} \mathrm{Used}_{-} \mathrm{cpu}_{\mathrm{ij}}$

un_mem $\mathrm{i}_{\mathrm{i}}$ is the amount of unused Memory in a host $\mathrm{i}$ and it is calculated as

un_mem $\mathrm{i}=$ Total_mem $\mathrm{i}-$ Static_mem ${ }_{\mathrm{i}}-\sum_{j=1}^{N}$ Used_mem $_{\mathrm{ij}}(\mathbf{3})$

un_disk $\mathrm{i}_{\mathrm{i}}$ is the amount of unused storage in a host $\mathrm{i}$ and it is calculated as

un_disk $k_{\mathrm{i}}=$ Total_disk $\mathrm{i}_{\mathrm{i}}-$ Static_disk $\mathrm{i}_{\mathrm{i}}-\sum_{j=1}^{N}$ Used_disk $_{\mathrm{ij}}$

And $r_{-} c u_{j}, r \_m e m_{j}, r \_d i s k_{j}$ is the requirement of incoming virtual machine in terms of number of processing cores, amount of memory and amount of storage disk respectively. Here $\mathrm{a}, \mathrm{b}, \mathrm{c}$ is the percentage of overall energy consumption donates by CPU, memory, storage disk. According to [19], the value of $\mathrm{a}$ is $58 \%, \mathrm{~b}$ is $28 \%$ and $\mathrm{c}$ is $14 \%$.

\section{Algorithm - VM consolidation with live migration}

1. Input: HostList, VMList, CurrentOnHostList

2. Output: VM is consolidated to the EnergyEfficientHost.

3. HostList $=$ GetTotalNoOfHost () ;

4. VMList $=$ GetTotalNoOfVM () ;

5. CurrentOnHostList $=$ GetCurrentNoOfOnHost();

6. EnergyEfficientHost = null;

7. EnoughHostList =null;

8. for each VM in VMList do

9. $\{$ if (CurrentOnHostList!=null)

10. \{ for each Host in CurrentOnHostList do

11. $\{$ if(Host.GetUnusedCPU $>=$ VM.GetRequiredCPU \&\& Host.GetUnuesdRam >= VM.GetRequiredRam\&\& Host.GetUnusedDisk >= VM.GetRequiredDisk) EnoughHostList.add(Host);

13. \} if(EnoughHostList!=null)

14. EnoughHostList.GetMinResources;

$$
\text { EnergyEfficientHost == }
$$

15.

HostWithHighMips();
16.

CurrentOnHostList.add(HostWithHighMips()); \}

17. \}

18. else $\{$ EnergyEfficientHost $=$ HostWithHighMips $($ );

19. CurrentOnHostList.add(HostWithHighMips());

20. VM is consolidated to the EnergyEfficientHost.

21.\}

22. VM optimization by VM migration.

23. Switch OFF idle node or the host with utilization $=0$;

24. end

When a user submits a request for allocating a VM, first of all, running hosts are traversed and check whether the available resources can satisfy the customer requirement or not. If more than one host can satisfy the request then we choose the host which consumes less power after allocating this VM, using equation (1). If remaining resources couldn't satisfy the incoming VM request, then new host having the highest capacity in terms of MIPS, will be turned-ON. During VM optimization, if the utilization of hosts goes below the utilization threshold, all VMs of that host will be migrated to the remaining switch ON hosts while satisfying the constraint that remaining host will not get over-utilized and then turn OFF the host with utilization value 0 . If the utilization of hosts goes above the utilization threshold value, then some VMs of over utilized host gets migrated to other host in order to reduce SLA violation.

\section{PERFORMANCE EVALUATION}

It is always difficult to perform experiments with different configurations, different requirements, and with different workload data, in real cloud environment. So there is a need of simulation framework for the performance evaluation of the proposed method. In the proposed work, we use CloudSim toolkit [8] as simulation framework which is built for simulation of Cloud computing environment. Other toolkits like SimGrid, GangSim are also available, but they did not support modeling of on-demand virtualization enabled resources. Cloudsim toolkit has the capability of modeling large scale computing environment including datacenters, physical machines and virtual machines. It has the classes to support simulation of a federated network with different network connection and network element. It also supports modeling of power consumption on datacenter.

For performance evaluation of our algorithm, we have taken a single Power aware datacenter having 10 Physical Machines (PM) with heterogeneous configuration of different types as shown in table 1 . The proposed method employs the assumption that a single user request can be served by a single VM. For experiment purpose we consolidate 51 virtual machines with different configuration.

Table 1: Different configuration of 10 Physical machines (PM)

\begin{tabular}{|c|c|c|c|}
\hline PM & CPU cores & Memory & Storage \\
\hline PM1 & 5 & $9740 \mathrm{MB}$ & $100 \mathrm{~GB}$ \\
\hline PM2 & 5 & $8096 \mathrm{MB}$ & $50 \mathrm{~GB}$ \\
\hline PM3 & 6 & $8096 \mathrm{MB}$ & $75 \mathrm{~GB}$ \\
\hline PM4 & 4 & $7096 \mathrm{MB}$ & $60 \mathrm{~GB}$ \\
\hline
\end{tabular}




\begin{tabular}{|c|c|c|c|}
\hline PM5 & 6 & $9500 \mathrm{MB}$ & $75 \mathrm{~GB}$ \\
\hline PM6 & 5 & $9740 \mathrm{MB}$ & $95 \mathrm{~GB}$ \\
\hline PM7 & 5 & $8096 \mathrm{MB}$ & $60 \mathrm{~GB}$ \\
\hline PM8 & 6 & $8096 \mathrm{MB}$ & $70 \mathrm{~GB}$ \\
\hline PM9 & 4 & $7096 \mathrm{MB}$ & $60 \mathrm{~GB}$ \\
\hline PM10 & 6 & $9500 \mathrm{MB}$ & $85 \mathrm{~GB}$ \\
\hline
\end{tabular}

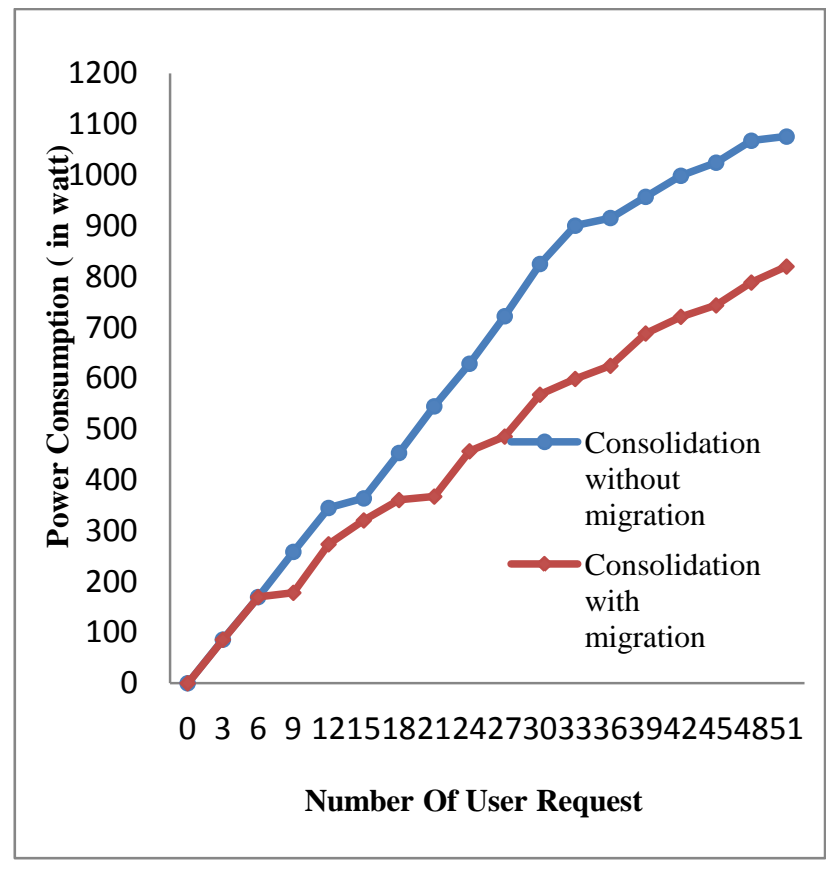

Fig. 3: Power Consumption comparison with different number of PMs

Fig. 3 provided the comparison of power comparison with different number of Physical Machines and interpreted that Power Consumption increases as the number of VMs increases, when employ the approaches. When VMs are consolidated without migration then it consumes more power as compared to consolidation with live migration. This is happened, because our proposed approach uses fewer physical machines i.e. minimum number of host is turned $\mathrm{ON}$ and save energy. Due to VM optimization in our proposed approach, the VMs hosted on the under loaded node gets migrated to the most power efficient node and switch OFF the under loaded host. For VM migration, we apply double threshold minimization of migration policy (DTMM). We compared DTMM with other policies described later. However due to migration the overhead can incur in terms of total time. But by limiting the number of migration the performance degradation will be minimized.

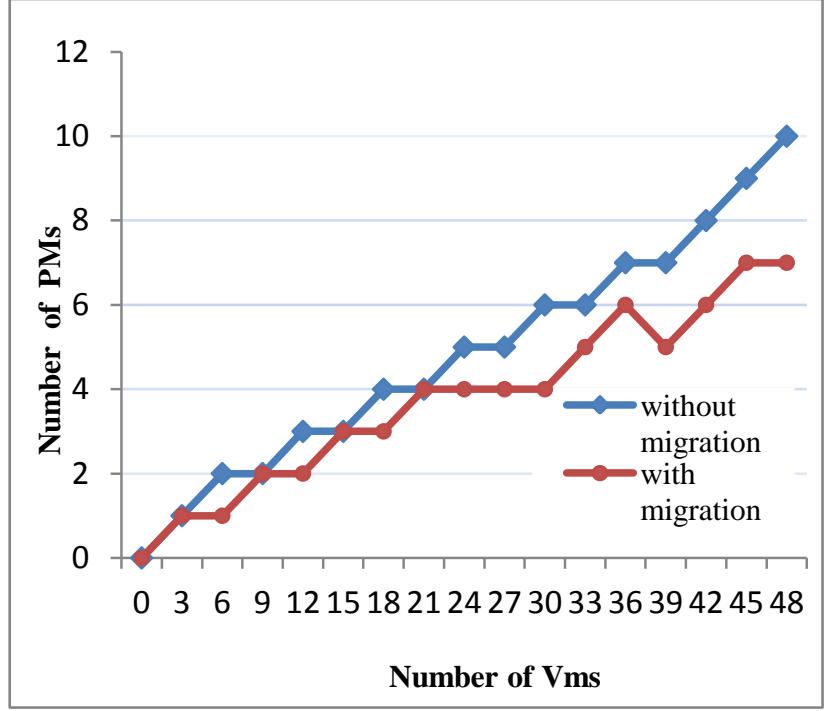

Fig. 4: Number of used PMs in different number of VMs

Fig. 4 Shows comparison of number of PMs used with number of VMs increases. The consolidation without migration approach uses more number of physical machines compared to our proposed method. When number of VMs is 45 and 48, the number of used PMs are stable i.e. 7 in case of consolidation with live migration. While in case of consolidation without migration, number of physical machine increases with number of virtual machine. This is because once a new host is power ON due to admission of new request, it remains $\mathrm{ON}$ whether it process a request or not and waste the valuable energy. On the other hand our proposed approach continuously optimizes VM and dynamically switches OFF host during overall processing.

We have compared Double Threshold Minimization of Migration (DTMM) policy with Static Threshold Minimum Migration Time (STMMT) policy with different thresholding values. The comparison in Fig. 5(a) shows that these migration policies approximately giving the same energy consumption. Whereas in Fig. 5(b) the number of VM migration produced by MM policy with threshold value 30$70 \%$ is minimum in comparison to Static threshold policy with 50 and $60 \%$ threshold value. MM policy with $30-70 \%$ is best for ensuring percentage of SLA violation of $1 \%$ as shown in Fig. 5(d) and average SLA violation with $6.7 \%$ comparatively with other policies in Fig. 5(c). Due to limited migrations, the overhead (in terms of migration time) is reduced. Thus MM policy can effectively balance the tradeoff between energy consumption and performance degradation. 


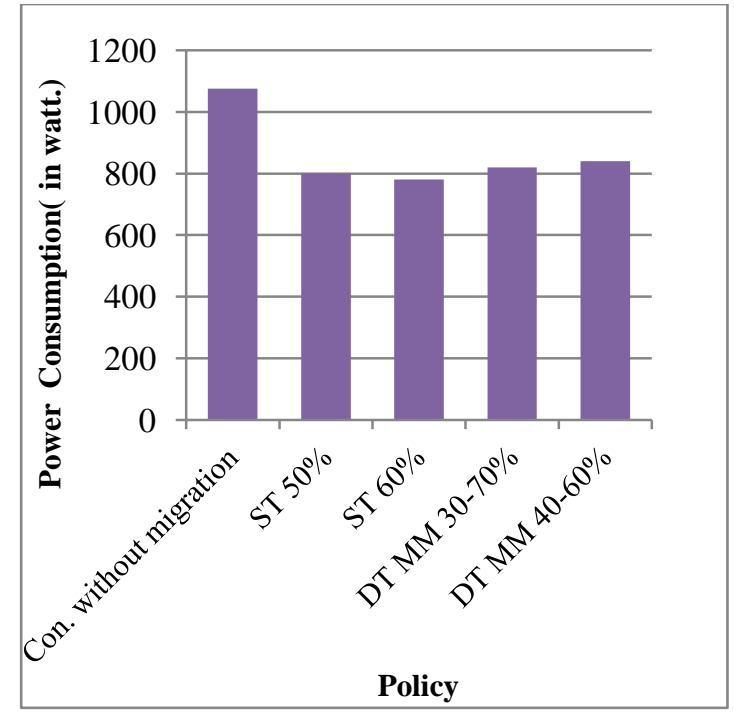

(a) Power Consumption

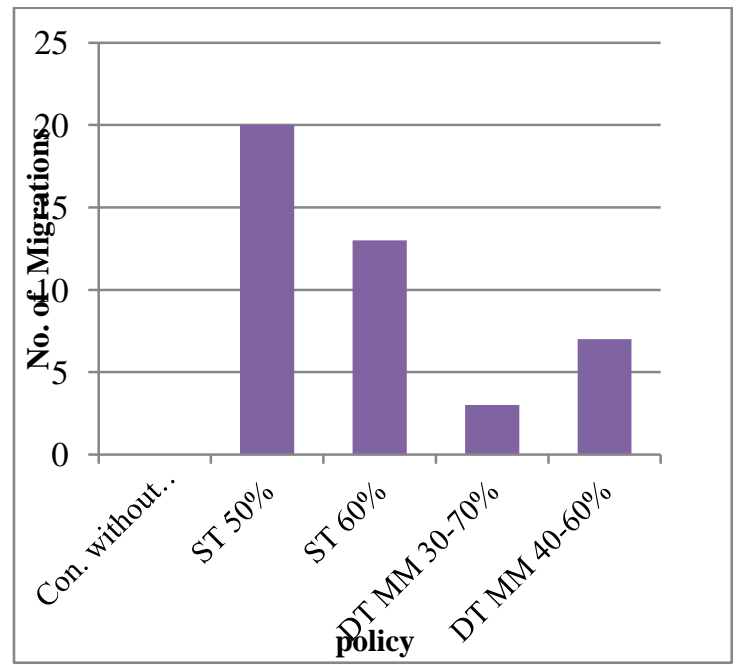

(b) No. of VM Migration

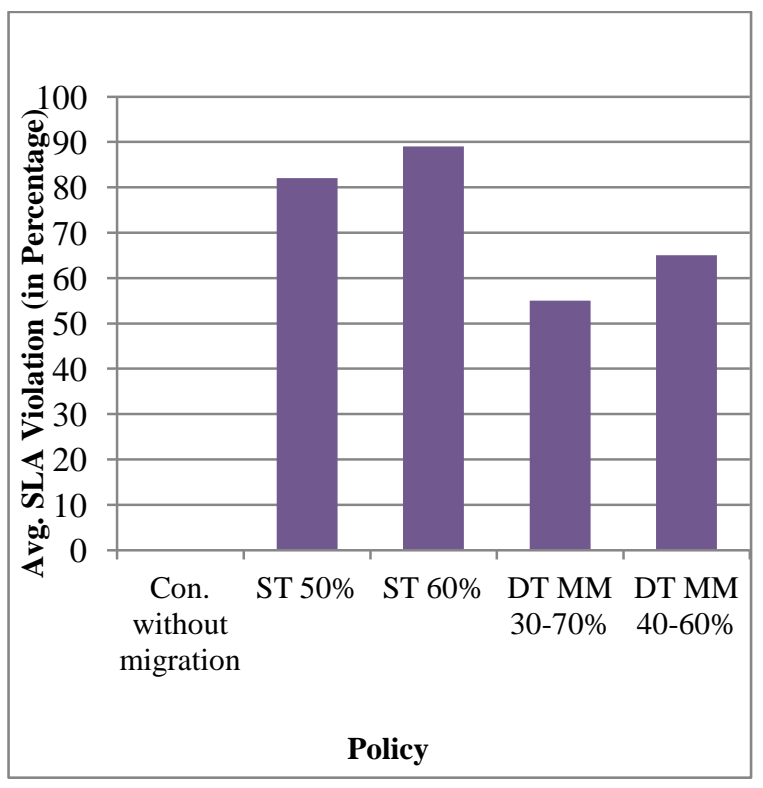

(c) Avg. SLA Violation

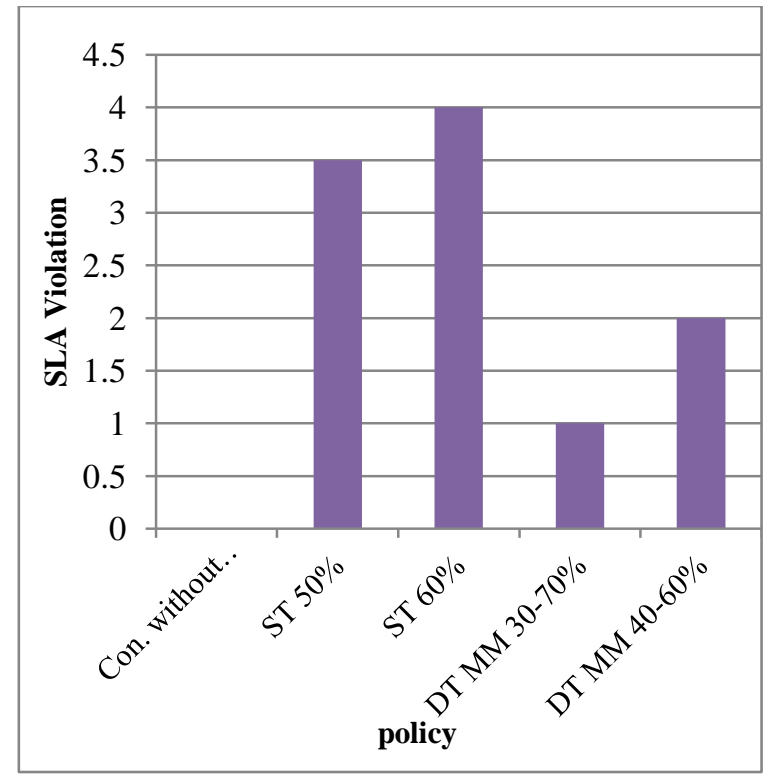

(d) SLA Violation

\section{CONCLUSION AND FUTURE WORK}

In this paper, we have presented a VM consolidation technique considering multiple resources with VM live migration in order to reduce the power consumption. Due to VM optimization in our proposed work, the VMs hosted on the under loaded node gets migrated to the most power efficient node and switch OFF the under loaded host. It is clear from the result that our approach is much energy efficient than other one and effectively maintained the tradeoff between energy consumption and performance degradation. We have shown that our approach could consolidate more number of VMs on to less number of hosts and tuned OFF maximum number of physical machines.

In the future work, it can be carrying out job prioritization with considering deadline. With this maximum number of user request could be satisfied within their deadline. We will also extend our work with adaptive threshold migration policy and investigate the algorithm behavior with multiple no. of resources on real cloud setup and check what will be exact energy consumption.

\section{REFERENCES}

[1] R. Yamini, "Power Management in Cloud Computing Using Green Algorithm", IEEE International Conference on Advances in Engineering, Science and Management (ICAESM), pp. 128-133, Mar. 2012.

[2] J.G. Koomey, Report on "Estimating total power consumption by servers in the U.S. and the world", Feb.5,2007.

http://sites.amd.com/de/Documents/svrpwrusecompletefi nal.pdf.

[3] V.K. Mohan Raj, R. Shriram, "Power Aware Provisioning in Cloud Computing Environment", IEEE International Conference on Computer, Communication and Electrical Technology (ICCCET), pp. 6-11, Mar. 2011. 
[4] Tiago C. Ferreto, Macro A.S. Netto, Rodrigo N. Calheiros, Cesar A.F. De Rose, "Server Consolidation with migration control for virtualized datacenters", ELSEVIER International journal on Future Generation Computer Science (FGCS), vol. 27, issue 8, pp.10271034, Oct. 2011.

[5] Anton Beloglazov and Rajkumar Buyya, "Energy Efficient Resource Management In Virtualized Cloud Datacenter", $10^{\text {th }}$ IEEE/ACM International Conference on Cluster, Cloud And Grid Computing (CCGRID), pp. 826-831, 2010.

[6] No Limits Software, David Cole's White Paper on "Datacenter Energy Efficiency - looking beyond PUE" http://www.globalknowledge.nl/content/files/documents/ 224536/Data-Center-White-Paper-Data-Center.

[7] M. Poess, R. Nambiar, "Energy cost, the key challenge of today's data centers: a power consumption analysis of TPC-C results", ACM Proceedings of the VLDB Endowment, vol. 1 issue 2, pp. 1229-1240, Aug. 2008.

[8] Rodrigo N. Calheiros, Rajiv Ranjan, César A. F. De Rose, and Rajkumar Buyya, "CloudSim: a toolkit for modeling and simulation of cloud computing environments and evaluation of resource provisioning algorithms", ACM journal of Software-Practice \& Experience, vol.41, issue 1, pp. 23-50, Jan. 2011.

[9] R. Neugebauer and D. McAuley, "Energy is just another re-source: Energy accounting and energy pricing in the nemesis OS", IEEE Proceedings of the Eighth Workshop on Hot Topics in Operating Systems, pp. 6772, May 2001

[10] H. Zeng, C. S. Ellis, A. R. Lebeck, and A. Vahdat, "ECOSys-tem: managing energy as a first class operating system resource," ACM SIGPLAN Notices, vol. 37, pp. 123-132, 2002

[11] E. Pinheiro, R. Bianchini, E. V. Carrera, and T. Heath, "Load balancing and unbalancing for power and performance in cluster-based systems", Workshop on Compilers and Operating Systems for Low Power, pp. 182-195, 2001

[12] Pat Bohrer, Elmootazbellah N. Elnozahy, Tom Keller, Michael Kistler, Charles Lefurgy, Chandler McDowell, and Ram Rajamony, "The case for power management in web servers", ACM Kluwer Academic Publishers, pp. 261-289, 2002

[13] Taliver Heath, Bruno Diniz, Wagner Meira Jr., Ricardo Bianchini, and Enrique V. Carrera, "Energy conservation in heterogeneous server clusters", $10^{\text {th }}$ ACM SIGPLAN Symposium on Principles and Practice of Parallel Programming (PPoPP05), pp. 186-195, 2005.

[14] F. Hermenier, X. Lorca, J. Menaud, G. Muller, J. Lawall, "Entropy: a consolidation manager for clusters", Proceedings of the ACM SIGPLAN/SIGOPS International Conference on Virtual Execution Environments, pp. 41-50, 2009.

[15] Y. Song, H. Wang, Y. Li, B. Feng, Y. Sun, "Multi-tiered on-demand resource scheduling for VM-Based data center", $9^{\text {th }}$ IEEE/ACM International Symposium on Cluster Computing and the Grid (CCGRID), pp. 148155, May 2009.

[16] L. Hu, H. Jin, X. Liao, X. Xiong, H. Liu, "Magnet: a novel scheduling policy for power reduction in cluster with virtual machines", IEEE International Conference on Cluster Computing, pp.13-22, Oct. 2008.

[17] E. Elnozahy, M. Kistler, R. Rajamony, "Energy-efficient server clusters, Power-Aware Computer Systems", ACM Proceedings of the $2^{\text {nd }}$ international conference on Power-aware computer systems (PACS'02), pp.179-197, 2003.

[18] M. Cardosa, M. Korupolu, A. Singh, "Shares and utilities based power consolidation in virtualized server environments" Proceedings of the $11^{\text {th }}$ IFIP/IEEE Integrated Network Management, IM, Long Island, NY, USA, pp. 327-334, 2009

[19] A. Kansal, F. Zhao, J. Liu, N. Kothari, and A.A. Bhattacharya, "Virtual machine power metering and provisioning", Proceedings of the $1^{\text {st }}$ ACM symposium on Cloud computing (SoCC), pp. 39-50, 2010.

[20] Jian-Sheng Liao, Chi-Chung Chang, Yao-Lun Hsu, XiaoWei Zhang, Kuan-Chou Lai, Ching-Hsien Hsu, "EnergyEfficient Resource Provisioning with SLA consideration on Cloud Computing”, $41^{\text {st }}$ IEEE International Conference on Parallel Processing Workshops (ICPPW) pp. 206-211, 2012 